\section{Antihistaminika bei nasaler Obstruktion}

A ntihistaminika spielen aufgrund ihrer blockierenden Wirkung auf die peripheren H1-Rezeptoren eine wichtige Rolle in der Therapie der saisonalen allergischen Rhinitis. Doch ist Allergie mehr als Histaminausschüttung - vor allem in der Spätphase spielen noch andere Mediatoren wie Leukotriene und Prostaglandine eine Rolle. Ein gutes Antihistaminikum sollte auch diese Allergiemediatoren bekämpfen.

Darüber hinaus sind weitere Forderungen an ein optimales Antiallergikum zu stellen: rascher Wirkungseintritt und langanhaltende Wirkung, antiobstruktive und antientzündliche Effekte, gleiche Wirksamkeit und Anwendbarkeit auch bei der perennialen Rhinitis, wenig unerwünschte Wirkungen.

Ein Therapeutikum, das diese Anforderungen weitgehend erfüllt, ist
Mizolastin. Dies wurde in mehreren klinischen Studien bestätigt. Die Wirkung setzt rasch ein, die therapeutische Sicherheit ist hoch, sedierende Effekte fehlen. Die prompte und umfassende Monotherapie mit Mizolastin ermöglicht in vielen Fällen eine Monotherapie, wobei die Wirksamkeit über 24 Stunden anhält.

Auch bei der perennialen Rhinitis erwies sich Mizolastin als gut wirksam und verbesserte die nasale Obstruktion. Dies ist insofern erwähnenswert, als viele Antihistaminika gegen das Symptom der nasalen Obstruktion eher schwach wirksam sind. In einer Studie an $48 \mathrm{Heu}$ schnupfenpatienten verbesserte Mizolastin jedoch in einer täglichen Dosierung von $10 \mathrm{mg}$ den nasalen Flow um mehr als 20\%. Dieser Wert entspricht jener Verbesserung, die auch von nasalen Steroiden her bekannt ist.

\title{
Asthma individuell therapieren
}

$\mathrm{D}$ em Asthma liegt eine chronische Entzündungsreaktion zugrunde, die zu einer Hyperreagibillität der Bronchien führt. Dadurch reagieren die Kinder auf Triggerfaktoren wie Allergene oder Luftverschmutzung mit der typischen Asthmasymptomatik. Neben Medikamenten, so C. Bauer, München, kommt deshalb präventiven Maßnahmen ein hoher Stellenwert zu.

Das bedeutet: Die Wohnungen der Asthmakinder sollten zur rauchfreien Zone erklärt werden. Liegt eine Milbenallergie vor, reduzieren Encasings die Asthmasymptomatik - klinisch relevant zum Teil erst nach acht Monaten.

Allergenreduktion allein reicht aber meist nicht aus. Schon bei Kindern mit einem Asthma vom Schweregrad 2 wird eine antientzündliche Dauertherapie notwendig. Zur Verfügung stehen Cromone bzw. Kortikosteroide. Gerade bei Kindern, so Bauer, sollte die Behandlung zunächst mit den gut verträglichen Cromonen begonnen werden.

Im Bedarfsfall werden Betamimetika, z.B. Reproterol, kurzfristig zusätz- lich zur Basismedikation gegeben. Braucht das Kind häufiger solche Reliever, ist der Einsatz eines gut vertäglichen Kombipräparates zu erwägen.

Indiziert ist das Kombinationspräparat nach Bauers Worten zwischen der Monotherapie mit Cromonen und der Dauertherapie mit Kortikoiden, also dann, wenn sich die Kinder zwischen der Asthma-Stufe 2 und 3 befinden.

Asthma vom Schweregrad 3 liegt vor, wenn das Kind mehr als einmal pro

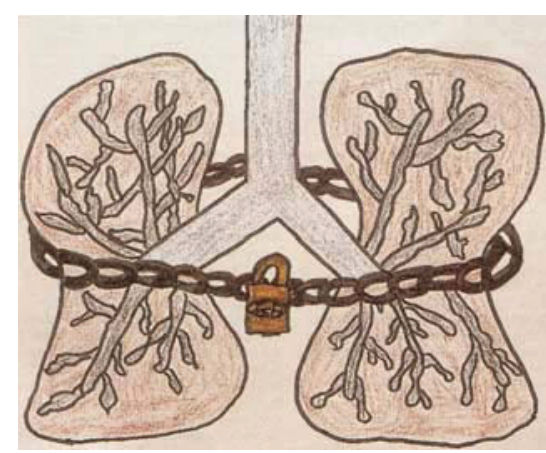

Wie Kinder Asthma erleben: Bild einer elfjährigen Patientin
Neben einer antihistaminergen Wirkung verfügt Mizolastin auch über antiinflammatorische Effekte z.B. im Sinne eines Leukotrienantagonismus: Durch die prophylaktische Gabe kann die Leukotrienausschüttung im Nasensekret unterdrückt werden - ein Befund der als Zeichen einer antientzündlichen Wirkung interpretiert werden kann.

Mit der hohen Wahrscheinlichkeit einer umfassenden Symptomreduktion auch im Rahmen einer Monotherapie mit Mizolastin erweist sich für den verordnenden Allergologen in Zeiten enger gefasster Budgetvorgaben eine solche Behandlungsstrategie in medizinischer als auch ökonomischer Hinsicht als effektiv.

$b k$

\section{Mösges R, Spaeth J, Tasman A-J.}

3. Zolim ${ }^{\circledR}$-Inselgespräch: „,Mizolastin mehr als nur Heuschnupfentherapie“, Westerland/Sylt 2001. Veranstalter: Schwarz Pharma Deutschland $\mathrm{GmbH}$.
Woche tagsüber und mehr als zweimal pro Monat nachts Beschwerden hat. Hier ist die tägliche Gabe von inhalativen Steroiden obligat, eine Cromontherapie nicht mehr ausreichend. Die Erfahrungen aus der Praxis zeigen, dass die meisten Kinder nicht kontinuierlich auf einem Schweregrad verharren. Vor allem bei Kleinkindem kann sich der Zustand unter einem Atemwegsinfekt im Winter verschlechtern oder das Asthma exazerbiert im Frühsommer bei Pollenflug.

Eine „Dauertherapie“ mit Entzündungshemmern ist also bei Kindern mit intermittierendem oder mildem persistierendem Asthma nicht immer über das gesamte Jahr notwendig. Ein solches Springen zwischen den Stufen kann eine psychologische Erleichterung für die Familien sein: Die Kinder sind nicht immer auf eine Dauertherapie z.B. mit Kortikoiden angewiesen. Wichtig ist, dass die Medikamente zur antientzündlichen Dauertherapie grundsätzlich über einen Spacer eingenommen werden, so Bauer.

red

Nach Informationen der Aventis Deutschland $\mathrm{GmbH}$ 